of order $m_{i}$, depends only on $\lambda_{i}$ and, by (4.6), its $(\alpha, \beta)$ element is a function of $\alpha-\beta$. If we define $N_{i}=\left(e_{2}, \cdots, e_{m_{i}}, 0\right)$, where $I=\left(e_{1}, \cdots, e_{m_{i}}\right)$, then

$$
L^{i i}=\sum_{\nu=0}^{m_{i}-1} \frac{p_{i}{ }^{(\nu)}\left(\lambda_{i}\right)}{\nu ! p_{i}\left(\lambda_{i}\right)} N_{i}{ }^{\nu}
$$

and is a polynomial in $N_{i}$. Since $J_{i}$ is also a polynomial in $N_{i}$ it must commute with $L^{i i}$.

The above results were derived for $H \in$ UHM. However, properties (ii) and (iii) generalize immediately to all Hessenberg matrices by the remarks at the beginning of Section 2.

University of California

Berkeley, California

1. J. G. F. Francis, "The $Q R$ transformation. I, II," Comput. J., v. 4, 1961/1962, pp. 265-271, 332-345. p. 20.

2. V. N. Faddeeva, Computational Methods of Linear Algebra, Dover, New York, 1959,

3. A. S. Householder, The Theory of Matrices in Numerical Analysis, Blaisdell, New York, 1964. MR 30 \#475.

4. M. Marcus \& H. Minc, A Survey of Matrix Theory and Matrix Inequalities, Allyn and Bacon, Boston, Mass., 1964. MR $29 * 112$.

5. J. H. Wilkinson, The Algebraic Eigenvalue Problem, Clarendon Press, Oxford, 1965. MR 32 * 1894 .

6. V. ZEIPEL, "On Determinates, hvars elementer aro Binomialkoefficenter," Lunds Univ. Asskr. II, 1865, pp. 1-68.

7. B. PARLETT, "Convergence theory for the $Q R$ algorithm on a Hessenberg matrix," Math. Comp. (To appear.)

\title{
An Elimination Method for Computing the Generalized Inverse*
}

\section{By Leopold B. Willner}

0. Notations. We denote by

$A \quad$ an $m \times n$ complex matrix,

$A^{*} \quad$ the conjugate transpose of $A$,

$A_{j}, j=1, \cdots, n$ the $j$ th column of $A$,

$A^{+} \quad$ the generalized inverse of $A$ [7],

$H$ the Hermite normal form of $A$, [6, pp. 34-36],

$Q^{-1} \quad$ the nonsingular matrix satisfying

$$
H=Q^{-1} A \text {, }
$$

$e_{i}, i=1, \cdots m$ the $i$ th unit vector $e_{i}=\left(\delta_{i j}\right)$,

$r \quad$ the $\operatorname{rank}$ of $A(=\operatorname{rank} H)$.

1. Method. The Hermite normal form of $A$ is written as

$$
H=\left[\begin{array}{l}
B \\
0
\end{array}\right] \quad \text { where } B \text { is } \quad r \times n .
$$

Received July 13, 1966.

* Research supported by the National Science Foundation Grant GP-5230. 
Combining (1) and (2) we have:

$$
A=Q H=[P, R] \quad\left[\begin{array}{l}
B \\
0
\end{array}\right]=P B,
$$

where $[P, R]$ is the corresponding partition of $Q$. Having displayed the $m \times n$ matrix $A$ of rank $r$ as a product of the $m \times r$ matrix $P$ and the $r \times n$ matrix $B$, which are both of rank $r$, we have as in [4]

$$
A^{+}=B^{+} P^{+}=B^{*}\left(B B^{*}\right)^{-1}\left(P^{*} P\right)^{-1} P^{*}
$$

therefore

$$
A^{+}=B^{*}\left(P^{*} P B B^{*}\right)^{-1} P^{*}
$$

and by $(3)$

$$
A^{+}=B^{*}\left(P^{*} A B^{*}\right)^{-1} P^{*} .
$$

The method can be summarized as follows:

Step 1. Given $A$ obtain $H$ by Gaussian elimination.

Step 2. From $H$ determine $P$ as follows:

The $i$ th column of $P, P_{i}, i=1, \cdots, r$ is

$$
P_{i}=A_{j} \quad \text { if } \quad H_{j}=e_{i}, \quad j=1, \cdots, n .
$$

Step 3. Calculate $P^{*} A B^{*}$.

Step 4. Invert $P^{*} A B^{*}$.

Step 5. Calculate $A^{+}$using (6).

2. Remarks. (i) From (7) we conclude that in order to obtain $P$ it is unnecessary to keep track of the elementary operations involved in finding $H$, e.g. [5].

(ii) Representation (4), as a computational method, was suggested by Greville [4], Householder [5] and Frame [2]. The novelty of the present paper lies in equation (6) and Step 2 above.

(iii) Like other elimination methods for computing $A^{+}$, e.g. [1], the method proposed here depends critically on the correct determination of rank $A$, e.g. the discussion in [3].

(iv) The advantage of method (6) over the elimination method of [1] is that here the matrix $A^{*} A$ ( or $A A^{*}$ ) is not computed. However, other matrix multiplications are involved in this method.

3. Example. For

$$
A=\left[\begin{array}{lllll}
0 & 1 & 0 & 1 & 1 \\
0 & 1 & 1 & 0 & 0 \\
0 & 2 & 1 & 1 & 1
\end{array}\right]
$$

we obtain by Gaussian elimination

$\left[\begin{array}{lllll}0 & 1 & 0 & 1 & 1 \\ 0 & 1 & 1 & 0 & 0 \\ 0 & 2 & 1 & 1 & 1\end{array}\right] \Rightarrow\left[\begin{array}{ccccc}0 & 1 & 0 & 1 & 1 \\ 0 & 0 & 1 & -1 & -1 \\ 0 & 0 & 1 & -1 & -1\end{array}\right] \Rightarrow\left[\begin{array}{ccccc}0 & 1 & 0 & 1 & 1 \\ 0 & 0 & 1 & -1 & -1 \\ 0 & 0 & 0 & 0 & 0\end{array}\right]=H$ since $H_{2}=e_{1}$ we have $P_{1}=A_{2}$, and since $H_{3}=e_{2}$ we have $P_{2}=A_{3}$. Hence for 
$A=P B$ we have

$$
\left[\begin{array}{lllll}
0 & 1 & 0 & 1 & 1 \\
0 & 1 & 1 & 0 & 0 \\
0 & 2 & 1 & 1 & 1
\end{array}\right]=\left[\begin{array}{ll}
1 & 0 \\
1 & 1 \\
2 & 1
\end{array}\right]\left[\begin{array}{ccccc}
0 & 1 & 0 & 1 & 1 \\
0 & 0 & 1 & -1 & -1
\end{array}\right]
$$

and

$$
P^{*} A B^{*}=\left[\begin{array}{cc}
12 & -3 \\
5 & 0
\end{array}\right]
$$

from which

$$
\left(P^{*} A B^{*}\right)^{-1}=\frac{1}{15}\left[\begin{array}{cc}
0 & 3 \\
-5 & 12
\end{array}\right]
$$

hence

$$
A^{+}=B^{*}\left(P^{*} A B^{*}\right)^{-1} P^{*}=\frac{1}{15}\left[\begin{array}{ccc}
0 & 0 & 0 \\
0 & 3 & 3 \\
-5 & 7 & 2 \\
5 & -4 & 1 \\
5 & -4 & 1
\end{array}\right] \text {. }
$$

Northwestern University

Evanston, Illinois 60201

1. A. Ben-Israel \& S. Wersan, "An elimination method for computing the generalized inverse of an arbitrary complex matrix," J. Assoc. Comput. Mach., v. 10, 1963, pp. 532-537. MR 31 *5318.

2. J. Frame, "Matrix functions and applications. I: Matrix operations and generalized inverses," IEEE Spectrum, v. 1, 1964, no. 3, pp. 209-220. MR 32 *1195.

3. G. GoluB \& W. KaHAN, "Calculating the singular values and pseudo-inverse of a matrix," J. SIAM Numer. Anal., v. 2, 1965, pp. 205-224.

4. T. N. E. GREville, "Some applications of the pseudo-inverse of a matrix," SIAM Rev., v. 2, 1960, pp. 15-22. MR 22 *1067.

5. A. S. HousenOLDER, The Theory of Matrices in Numerical Analysis, Blaisdell, New York, 1964 . MR 30 *5475.

6. M. Marcus \& H. Minc, $A$ Survey of Matrix Theory and Matrix Inequalities, Allyn and Bacon, Boston, Mass., 1964. MR 29 * 112.

7. R. PENROSE, “A generalized inverse for matrices," Proc. Cambridge Philos. Soc., v. 51, 1955, pp. 406-413. MR 16, 1082. 